\title{
Precoding for the Multiantenna Downlink: Multiuser SNR Gap and Optimal User Ordering
}

\author{
Chi-Hang Fred Fung, Student Member, IEEE, Wei Yu, Member, IEEE, and Teng Joon Lim, Senior Member, IEEE
}

\begin{abstract}
This paper develops a practical design method for implementing Tomlinson-Harashima precoding (THP) in a downlink channel with multiple antennas at the transmitter and a single antenna at each receiver. A two-step design process is proposed for minimizing the total transmit power while satisfying every user's minimum data rate and maximum bit-error rate (BER) requirements. First, the BER and rate requirements are converted to "virtual rate" requirements, which account for the gap-to-capacity introduced by practical quadrature amplitude modulation (QAM) and THP. The second step is to determine the transmit covariance matrices (which specify the entire THP system) that will provide these virtual rates at the minimum total transmit power. As one of the main features in the proposed scheme, an algorithm for finding the optimal user encoding (or presubtraction) order in polynomial time is proposed. In addition, we also propose an algorithm that finds a near-optimal order, but which is much less complex. The proposed method outperforms existing zero-forcingbased THP systems in term of power efficiency.
\end{abstract}

Index Terms-Broadcast channel (BC), multiple-access channel (MAC), multiple antennas, signal-to-noise ratio (SNR) gap approximation, spatial multiplex, Tomlinson-Harashima precoding (THP), uplink-downlink duality.

\section{INTRODUCTION}

$\mathbf{I}$ N FIXED wireless networks, such as broadband wireless access (BWA) and wireless local area networks (WLANs), it is feasible and desirable to move most of the signal processing complexity from the subscriber stations to the base station. This so-called transmitter precoding strategy reduces complexity in the subscriber stations, which subsequently become cheaper and more power-efficient. The practicality of precoding is founded on the feasibility of feeding back channel estimates from the subscriber stations to the base station when the channels are essentially time-invariant. The availability of such a channel estimation and feedback method is assumed for the rest of this paper.

In this paper, we are concerned with optimal transmitter design for a downlink channel with one transmitter and several receivers, which is also known as a broadcast channel (BC) in the

\footnotetext{
Paper approved by M. Chiani, the Editor for Transmission Systems. Manuscript received June 14, 2005; revised January 15, 2006. This work was supported by the Natural Science and Engineering Research Council (NSERC) of Canada. This work was presented in part at the 22nd Biennial Symposium on Communications, Queen's University, Kingston, ON, Canada, May 2004, and in part at the IEEE Singapore International Conference on Communication Systems (ICCS), Singapore, September 2004.

The authors are with the Edward S. Rogers Sr. Department of Electrical and Computer Engineering, University of Toronto, Toronto, ON M5S 3G4, Canada (e-mail: cffung@comm.utoronto.ca; weiyu@comm.utoronto.ca; tj.lim@utoronto.ca).

Digital Object Identifier 10.1109/TCOMM.2006.885095
}

information theory literature. The transmitted signal is assumed to have multiple signal dimensions, and linear filtering of the signal (which corresponds to beamforming if the signal dimensions are spatial) and interference precancellation at the transmitter are performed. The received signals are one-dimensional (corresponding, for instance, to the case where the receiver is equipped with only one antenna). This is a system setup of considerable practical importance, since many portable devices have room for only one antenna. As well, many devices have a matched filter or diversity-combining front-end that reduces the received signal into a scalar decision statistic in each symbol interval. The latter scenario is mathematically equivalent to the single-receive-antenna scenario.

Much work has been reported in the literature on the topic of precoding for the downlink channel. Precoder designs for the downlink can be divided into linear and nonlinear methods, and further into interference-suppression approaches and signal-tointerference-and-noise ratio (SINR) constrained ones. Linear interference-suppression techniques (see e.g., [1] and [2]) refer to the use of transmit beamforming weights for each user that result in no multiuser interference (MUI) at each receiver. Basically, this is zero-forcing (ZF) interference suppression applied at the transmitter rather than the receiver, and it suffers from the same problems as ZF receivers, e.g., noise or power enhancement and a hard limit on the number of interference sources that can be dealt with.

This paper focuses on nonlinear schemes in which a matrix form of the Tomlinson-Harashima precoder can be implemented at the transmitter to successively precancel MUI (see e.g., [3] and [4]). Transmitter-based Tomlinson-Harashima precoding (THP) is equivalent to decision-feedback equalization (DFE) at the receiver. THP can be designed with a ZF criterion, in which case the channel matrix is triangularized by a $\mathrm{QR}$ factorization, and individual user SINRs may be guaranteed by an additional power-loading step [3], [4]. However, such a design is not optimum, because the THP structure is a function of the channel matrix only and not of individual user SINR requirements. ${ }^{1}$

A more powerful approach discards the notion of interference suppression as a design objective, and instead asks the question: How do we satisfy individual SINR requirements with minimal transmitted power? This problem has essentially been solved for the case where each receiver has only one antenna, both using the linear (purely beamforming) formulation [5]-[7], and recently with nonlinear successive "dirty-paper encoding" with a fixed encoding order [8]. When each receiver

\footnotetext{
${ }^{1}$ Alternatively, a minimum mean-squared error (MMSE) criterion can be used for THP design as well. However, conventional MMSE design also does not account for individual user data-rate requirements directly.
} 
is allowed to have more than one antenna, several suboptimal approaches have been introduced (e.g., [9] and [10] describe linear approaches; [11] describes a nonlinear method).

Key to the single-antenna solutions is an uplink-downlink SINR duality theorem that states that if a set of SINRs is achievable on the uplink, then it is also achievable on the downlink under the same total power constraint (and vice versa) for both linear [5]-[7] and nonlinear [8] precoding systems. ${ }^{2}$ In the case of nonlinear precoding, the dual uplink channel implements successive decoding or interference cancellation at the receiver, with an order of detection that is the reverse of the encoding or presubtraction order for the downlink. Furthermore, for the case of single-antenna subscriber stations, the MMSE receive beamformers for the virtual uplink are exactly the transmit beamformers for the downlink that will produce the same SINRs. This concept of duality is powerful, because the optimal uplink transmission problem is much easier to solve than the corresponding downlink problem.

However, SINR duality simply states that the set of achievable SINRs on the downlink and uplink are identical, for a given downlink encoding order. Each encoding order has its own achievable SINR region. Therefore, truly minimizing total power while satisfying individual SINR constraints requires us to design optimal transmitters for each of $K$ ! encoding orders, where $K$ is the number of users, then to pick the one which uses least power. This exhaustive search approach is necessitated by the absence of a general theory on the geometry of achievable SINR regions. We observe in this paper that such a theory is instead available from an information-theoretical capacity region point of view. Consequently, the user-ordering problem can be solved efficiently without exhaustive search if we impose rate constraints rather than SINR constraints, and make use of the notion of capacity-region duality.

Capacity-region duality refers to the equivalence of the achievable rate regions of the multiple-access channel (MAC) and the $\mathrm{BC}$ under a sum-power constraint, first established in [12] and [13]. Although obtained independently, this notion of duality bears a striking resemblance to the SINR-based duality result with successive decoding/encoding [8]. This is because in the special case of only one antenna at the subscriber station, achievable rates are monotonically related to SINR. However, the two approaches also differ in two respects. On the one hand, the SINR duality is more practically useful, as SINR is a concept that has links to practical notions of data rate and bit-error probability (BEP) (assuming that only one symbol stream is being transmitted to or from each user). On the other hand, the capacity-region duality result is more powerful, in that the user encoding order at each point on the boundary of the region is known, and there are no restrictions on the number of antennas at each subscriber station. The former point will be explained in Section III-B and used in Section IV-B.

The main contribution of this paper is the use of capacityregion duality to solve the optimal precoder design and optimal user-ordering problems efficiently for systems with individual

\footnotetext{
${ }^{2}$ The equivalent SINR duality result is unavailable for the downlink channel with multiantenna receivers, because then multiple data streams can be transmitted to each user, in which case SINR is not well-defined. But if each user is constrained to only one data stream, then duality applies as shown in [11].
}

rate and bit-error rate (BER) requirements. Toward this end, we demonstrate the following.

1) The concept of the signal-to-noise ratio (SNR) gap, traditionally used to relate SNR and capacity for practical quadrature amplitude modulated (QAM) single-user systems with a BER requirement can be generalized to systems that implement MUI precancellation schemes based on THP.

2) The gap in SINR can be approximated by a gap in capacity. This allows the notion of "virtual rate region" to be defined and exploited in the proposed solution.

3) The optimal encoding order problem for a nonlinear THPbased precoding system that minimizes transmitted power while satisfying BEP and data-rate requirements can be solved with a complexity that is polynomial in $K$, the number of users.

4) A low-complexity, near-optimal user-ordering algorithm can be found with even lower complexity.

The rest of the paper is organized as follows. In Section II, we describe the problem formulation and present the concept of virtual rates, which allows capacity-region duality to be used in a practical setting. Section III contains an overview of relevant results in multiuser information theory. Section IV contains the main result of the paper, which is an optimal user-ordering scheme for a nonlinearly precoded downlink, and a near-optimal low-complexity alternative. Section V contains simulation results, and Section VI concludes the paper.

\section{PROBLEM Formulation}

\section{A. Signal Model}

Consider a $K$-user downlink system with $t$ transmit dimensions at the base station and one receive dimension at each remote user. The $i$ th user's receiver generates the scalar sufficient statistic

$$
y_{i}=\mathbf{h}_{i}^{T} \mathbf{x}+z_{i}, \quad i=1, \ldots, K
$$

where $\mathbf{h}_{i} \in \mathbb{C}^{t \times 1}$ represents the channel, $\mathbf{x} \in \mathbb{C}^{t \times 1}$ is the transmitted signal vector, and $z_{i}$ is a circularly symmetric Gaussian random variable with zero mean and unit variance. The signal $\mathbf{x}$ is generated from all symbols $\left\{u_{1}, \ldots, u_{K}\right\}$ intended for users $1 \ldots K$, and, therefore, $y_{i}$ in general contains MUI. This model can be used with multiantenna base stations with nondispersive (frequency-flat) channels; it is equally applicable to synchronous code-division multiaccess (CDMA) systems in which $\mathbf{h}_{i}$ is a vector of so-called code correlations between the $i$ th spreading code and all codes in use, including itself. In this paper, we adopt the former interpretation for convenience and without loss of generality. The design objective is to provide user $i$ with a given data rate $R_{i}$ and a given maximum BER while minimizing the total transmit power.

Before treating the practical design problem as stated above, it is useful to examine the theoretical limits of this downlink channel. From information-theoretical considerations, the capacity region of a downlink channel is achieved with the so-called dirty-paper coding (DPC) technique [3], [12]-[17], in which the transmitter uses a nonlinear successive precancellation scheme to mitigate MUI seen at the receiver. More 
specifically, fix an encoding order $\boldsymbol{\pi}$ in which user $\pi(K)$ is encoded first and user $\pi(1)$ is encoded last. Let $\mathbf{S}_{i}$ be the transmit covariance matrix for user $i$. The following rate is achievable by user $\pi(i)$ :

$$
R_{\pi(i)}^{\mathrm{BC}}(\boldsymbol{\pi}, \mathcal{S})=\log \left(1+\frac{\mathbf{h}_{\pi(i)}^{H} \mathbf{S}_{\pi(i)} \mathbf{h}_{\pi(i)}}{1+\mathbf{h}_{\pi(i)}^{H} \sum_{j=1}^{i-1} \mathbf{S}_{\pi(j)} \mathbf{h}_{\pi(i)}}\right)
$$

where $\mathcal{S}=\left\{\mathbf{S}_{1}, \ldots, \mathbf{S}_{K}\right\}$ is the set of all transmit covariance matrices [16].

In a DPC system, users $\pi(i+1)$ through $\pi(K)$ are treated as known interference and, therefore, by the DPC theorem of [14] do not affect the capacity of user $\pi(i)$. Users $\pi(1)$ through $\pi(i-1)$, on the other hand, are not yet encoded, and, hence, must be treated as unknown interference. As the optimal input distributions are all Gaussian and the noise variance is unity, the expression above follows.

Note that the above expression assumes that the interference is Gaussian-like. This can be justified via the central limit theorem in the limit of large numbers of interfering users, or by noting that the above expression is a lower bound on the actual achievable rate, as Gaussian noise is the worst possible noise subject to a covariance constraint.

The objective of the downlink design problem is to minimize the total transmit power over all transmit covariance matrices $\mathbf{S}_{i}$ and all possible encoding orders $\boldsymbol{\pi}$, subject to a target rate constraint

$$
\min _{\mathcal{S}, \boldsymbol{\pi}} P \quad \text { s.t. } \quad \mathbf{R}^{\text {target }} \in \mathcal{C}_{\mathrm{BC}}(P, \mathbf{H})
$$

where the total transmit power is $P=\sum_{i} \operatorname{tr}\left(\mathbf{S}_{i}\right), \mathbf{R}^{\text {target }}$ is the target rate-tuple, and $\mathcal{C}_{\mathrm{BC}}(P, \mathbf{H})$ denotes the capacity region of the $\mathbf{B C} \mathbf{H}=\left[\mathbf{h}_{1}, \ldots, \mathbf{h}_{K}\right]^{T}$ under a total power constraint $P$, i.e., the set of rates $\left(R_{1}, \ldots, R_{K}\right)$ which can be achieved in this channel with maximum total power $P$.

The dirty-paper encoder has the effect of precancelling an interference term $\mathbf{h}_{\pi(i)}^{H} \sum_{j=i+1}^{K} \mathbf{S}_{\pi(j)} \mathbf{h}_{\pi(i)}$ that would otherwise have been a part of the noise. Clearly, this information-theoretical limit is achievable only if capacity-achieving codes are used. In this paper, we adopt THP [18], [19] as a practical realization of DPC [3], [15], [20]. (See [21] for a comprehensive introduction.) Our implementation of THP as a MUI presubtraction scheme is described in more detail in Section IV-A.

Note that both DPC and THP use coset coding methods, i.e., each information symbol is represented by a large set of codewords (DPC) or signals (THP). The codeword or signal actually transmitted depends on the interference encountered as well as the symbol to be transmitted. However, practical THP incurs a finite loss as compared with ideal DPC. Thus, for practical systems, the optimization problem (3) needs to be modified to take the loss into account.

\section{B. SNR Gap-to-Capacity}

The capacity of an additive white Gaussian noise (AWGN) channel with an SNR $\gamma$ is

$$
C=\log (1+\gamma) .
$$

In practice, QAM constellations are used to approach the AWGN channel capacity in the moderate- and high-rate regimes $(C \geq 1)$. However, uncoded QAM is far from capacity-achieving. To achieve a given data rate at a given probability of error with QAM, a value of SNR higher than that suggested by (4) is required. This extra amount of power is called the SNR gap, denoted in this paper as $\Gamma_{\text {mod }}$.

The SNR gap is a useful concept, because it is roughly independent of the constellation size for square QAM, and is easily computable as a function of the probability of error target [22], [23]. (At $P_{e}=10^{-6}, \Gamma_{\mathrm{mod}}=8.8 \mathrm{~dB}$. At $P_{e}=10^{-7}, \Gamma_{\mathrm{mod}}=$ $9.5 \mathrm{~dB}$.) The definition of $\Gamma_{\text {mod }}$ allows the computation of the achievable rate for a practical QAM constellation in AWGN as

$$
R=\log \left(1+\frac{\gamma}{\Gamma_{\bmod }}\right) .
$$

Note that $\Gamma_{\text {mod }}$ may be reduced by the use of error-correcting codes.

This paper generalizes the above concept to multiuser downlink channels. Toward this end, we first note that the concept of the SNR gap can be readily extended to include THP systems with QAM. As compared with QAM on an AWGN channel, THP incurs additional losses due to power amplification and an increase in the number of nearest neighbors for constellation points on the perimeter of the constellation. Such losses can be accurately characterized [20], [24] and are denoted as $\Gamma_{\mathrm{TH}}$ in this paper. Together, $\Gamma_{\mathrm{mod}}$ and $\Gamma_{\mathrm{TH}}$ modify the achievable rate expression from $\log (1+\gamma)$ to

$$
R=\log \left(1+\frac{\gamma}{\Gamma_{\bmod } \Gamma_{\mathrm{TH}}}\right) .
$$

Now, consider a multiuser downlink channel with a set of achievable rates given by (2). To take into account practical QAM and THP, the required SINR must be increased by a factor $\Gamma_{\bmod } \Gamma_{\mathrm{TH}}$. Thus, the achievable rate for user $\pi(i)$ becomes

$$
\begin{aligned}
& R_{\pi(i)}^{\mathrm{BC}}\left(\boldsymbol{\pi}, \mathcal{S}, \Gamma_{\mathrm{mod}}, \Gamma_{\mathrm{TH}}\right) \\
& =\log \left(1+\frac{\mathbf{h}_{\pi(i)}^{H} \mathbf{S}_{\pi(i)} \mathbf{h}_{\pi(i)}}{\Gamma_{\mathrm{mod}} \Gamma_{\mathrm{TH}}\left(1+\mathbf{h}_{\pi(i)}^{H} \sum_{j=1}^{i-1} \mathbf{S}_{\pi(j)} \mathbf{h}_{\pi(i)}\right)}\right) .
\end{aligned}
$$

The downlink power-minimization problem with the SNR gap to capacity accounted for can be described as

$$
\min _{\mathcal{S}, \boldsymbol{\pi}} P \quad \text { s.t. } \quad \mathbf{R}^{\text {target }} \in \mathcal{R}_{\mathrm{BC}}\left(P, \mathbf{H}, \Gamma_{\text {mod }}, \Gamma_{\mathrm{TH}}\right)
$$

where again $P=\sum_{i} \operatorname{tr}\left(\mathbf{S}_{i}\right), \mathbf{R}^{\text {target }}$ is the target rate-tuple, and $\mathcal{R}_{\mathrm{BC}}\left(P, \mathbf{H}, \Gamma_{\text {mod }}, \Gamma_{\mathrm{TH}}\right)$ denotes the set of rates achievable with SNR gaps of $\Gamma_{\bmod }$ and $\Gamma_{\mathrm{TH}}$ due to practical modulation and THP, respectively.

\section{Gap and Duality}

Interestingly, and perhaps surprisingly, the inclusion of the $\Gamma_{\text {mod }} \Gamma_{\mathrm{TH}}$ term makes (8) a much more difficult problem than (3). Although neither of the rate expressions (2) and (7) is a convex function of $\mathbf{S}_{\pi(j)}$, the optimization problem (3) can be converted into a convex one via a set of capacity-region duality transformations between the $\mathrm{BC}$ and the MAC, as illustrated in [12], [13], and [25]. In this case, the optimal covariance matrices 
that correspond to each point on the boundary of the capacity region can be computed via convex optimization methods. However, this duality is a happy coincidence in information theory, and the duality transformation does not hold for (7) when practical coding and modulation schemes are employed. The use of a practical modulation scheme on the $\mathrm{BC}$ calls for new methods of optimization.

\section{ViRTUAL UPLINK-DOWNLINK DUALITY}

\section{A. Virtual Rate}

At first glance, one might suspect that the optimal downlink design for a practical system can be derived by designing an ideal capacity-achieving set of transmit covariance matrices $\left\{\mathbf{S}_{i}\right\}_{i=1}^{K}$ first, then scaling each $\mathbf{S}_{i}$ by a factor $\Gamma_{\text {mod }} \Gamma_{\mathrm{TH}}$. This is, however, not the case, as doing so would also increase interference power. Thus, it does not have the effect of increasing SINR by the same factor. In fact, the information-theoretical method of capacity-region duality, which converts a difficult nonconvex downlink problem to a convex uplink problem, breaks down whenever $\Gamma_{\text {mod }} \Gamma_{\mathrm{TH}} \neq 0 \mathrm{~dB}$.

Alternatively, if we knew the optimal encoding order $\pi_{0}$, we could use SINR duality with each user's SINR requirement scaled up by $\Gamma_{\bmod } \Gamma_{\mathrm{TH}}$ [8]. But the solution of (8) requires optimization over $\boldsymbol{\pi}$ as well as $\left\{\mathbf{S}_{1}, \ldots, \mathbf{S}_{K}\right\}$, and the achievable SINR region under a total power constraint does not have a structure that allows one to derive an efficient user-ordering technique. This is in contrast to working with the $\mathrm{BC}$ capacity region (or equivalently, the MAC capacity region), which is the convex hull of polymatroids and has a geometric structure that may be exploited to solve both the user-ordering problem and covariance matrix computation problem within (8).

The key in the formulation of our solution is a derivation of a gap in rate from the gap in SNR. Assuming desired rates above a moderate value (i.e., $R \geq 1$ ), the rate expression (6) can be approximated as

$$
R \approx \log (1+\gamma)-\log \left(\Gamma_{\bmod } \Gamma_{\mathrm{TH}}\right) .
$$

In other words, at moderate and high SINR, the achievable rate with SINR equal to $\gamma$ is approximately equal to the actual capacity minus a fixed constant, which depends on the modulation format and the target BER. Writing it in another way, we may define a "virtual rate"

$$
\tilde{R}=R+\log \left(\Gamma_{\bmod } \Gamma_{\mathrm{TH}}\right) \approx \log (1+\gamma) .
$$

This expression states that for a desired rate $R$ and a desired BER, the required SINR can be computed as $2^{\tilde{R}-1}$. It also says that if we design a system that yields rates of $\tilde{R}$ from an information-theoretic standpoint, it will satisfy the original rate and BER requirements with uncoded QAM and practical THP.

The point of defining virtual rates $\tilde{R}$ based on the BER and rate requirements is that a virtual rate-region duality can then be used to significantly simplify the downlink transmit-power minimization problem, which can now be stated as follows:

$$
\min _{\mathcal{S}, \boldsymbol{\pi}} P \quad \text { s.t. } \quad \tilde{\mathbf{R}}^{\text {target }} \in \mathcal{C}_{\mathrm{BC}}(P, \mathbf{H})
$$

where

$$
\begin{aligned}
\tilde{\mathbf{R}}^{\text {target }} & =\left[\tilde{R}_{1}^{\text {target }}, \ldots, \tilde{R}_{K}^{\text {target }}\right] \text { and } \\
\tilde{R}_{i}^{\text {target }} & =R_{i}^{\text {target }}+\log \left(\Gamma_{\text {mod }} \Gamma_{\mathrm{TH}}\right) .
\end{aligned}
$$

Essentially, the $\Gamma_{\bmod } \Gamma_{\mathrm{TH}}$ term in (8) is now moved from the SNR expression to the rate expression. Doing so allows an uplink-downlink duality result for the capacity region to be used.

\section{B. Uplink-Downlink Duality for Capacity Regions}

As explained earlier, the capacity region of a BC is achieved with successive dirty-paper precoding. For a given encoding order $\pi(K) \rightarrow \pi(1)$ and transmit covariance matrices $\mathbf{S}_{k}, k=$ $1, \ldots, K$, the set of rates achievable is

$$
\mathbf{R}^{\mathrm{BC}}(\boldsymbol{\pi}, \mathcal{S})=\left[R_{1}^{\mathrm{BC}}(\boldsymbol{\pi}, \mathcal{S}), \ldots, R_{K}^{\mathrm{BC}}(\boldsymbol{\pi}, \mathcal{S})\right]
$$

where $R_{k}^{\mathrm{BC}}(\pi, \mathcal{S})$ is obtained from (2).

The capacity region $\mathcal{C}_{\mathrm{BC}}(P, \mathbf{H})$ is the convex hull of the union of all rate vectors obtained from successive encoding over all encoding orders $\pi$ and all transmit covariance matrices that satisfy the sum power constraint $\sum_{i=1}^{K} \operatorname{tr}\left(\mathbf{S}_{i}\right) \leq P$

$$
\mathcal{C}_{\mathrm{BC}}(P, \mathbf{H})=\operatorname{Co}\left(\bigcup_{\boldsymbol{\pi}, \mathcal{S}} \mathbf{R}^{\mathrm{BC}}(\boldsymbol{\pi}, \mathcal{S})\right)
$$

where $\mathrm{Co}(\cdot)$ denotes the "convex hull" operation, all $\mathbf{S}_{i}$ must be positive semidefinite, and $\sum_{i=1}^{K} \operatorname{tr}\left(\mathbf{S}_{i}\right) \leq P$.

The problem (11) is equivalent to finding a power constraint $P$ such that $\tilde{\mathbf{R}}^{\text {target }}$ is on the boundary of $\mathcal{C}_{\mathrm{BC}}(P, \mathbf{H})$, but this reformulation is not helpful because the boundary cannot be found easily. The difficulty primarily lies in the fact that the weighted sum of BC rates is a nonconvex function of $\mathbf{S}_{i}$. However, as mentioned before, a capacity-region duality result can significantly simplify the problem. It has been shown [12], [13] that $\mathcal{C}_{\mathrm{BC}}(P, \mathbf{H})=\mathcal{C}_{\mathrm{MAC}}\left(P, \mathbf{H}^{H}\right)$ where $\mathcal{C}_{\mathrm{MAC}}$ refers to the capacity region of a MAC with a sum-power constraint $P$ and received signal

$$
\mathbf{y}=\sum_{i=1}^{K} \mathbf{h}_{i} d_{i}+\mathbf{n}
$$

where $\mathbf{h}_{i}$ is precisely the $i$ th channel vector on the downlink, $d_{i}$ is the scalar signal transmitted by the $i$ th user, and $\mathbf{n}$ is additive Gaussian noise with covariance matrix $\mathbf{I}$. The sum-power constraint is given by $\sum_{i=1}^{K} \mathbb{E}\left|d_{i}\right|^{2} \leq P$.

A transformation that depends solely on the channel matrix $\mathbf{H}$ and noise statistics can be defined to map every set of downlink transmit covariance matrices $\left\{\mathbf{S}_{i}\right\}$ to a set of virtual uplink transmit variances $\left\{q_{i}\right\}$, where $q_{i}=\mathbb{E}\left|d_{i}\right|^{2}$, and vice versa, so that the same set of rates is achievable on the downlink and its virtual uplink. The encoding order of the former is the reverse of the decoding order of the latter, i.e., on the virtual uplink, user $\pi(1)$ is decoded first and $\pi(K)$ last.

The achievable rate vector on the virtual uplink is

$\mathbf{R}^{\mathrm{MAC}}\left(\boldsymbol{\pi},\left\{q_{i}\right\}\right)=\left\{\log \frac{\left|\mathbf{I}+\sum_{j=i}^{K} q_{\pi(j)} \mathbf{h}_{\pi(j)} \mathbf{h}_{\pi(j)}^{H}\right|}{\left|\mathbf{I}+\sum_{j=i+1}^{K} q_{\pi(j)} \mathbf{h}_{\pi(j)} \mathbf{h}_{\pi(j)}^{H}\right|}\right\}_{i=1}^{K}$ 
which can be derived from first principles, treating users $\pi(1)$ through $\pi(i-1)$ as perfectly cancelled and the rest as unknown interferers. Similar to the BC capacity region, the MAC capacity region with sum-power constraint $P$ is

$$
\mathcal{C}_{\mathrm{MAC}}\left(P, \mathbf{H}^{H}\right)=\mathrm{Co}\left(\bigcup_{\boldsymbol{\pi},\left\{q_{i}\right\}} \mathbf{R}^{\mathrm{MAC}}\left(\boldsymbol{\pi},\left\{q_{i}\right\}\right)\right)
$$

where $\sum_{i} q_{i} \leq P$. The problem (11) is now equivalent to finding $\left\{q_{i}\right\}$ (and, hence, $P$ ), such that the desired virtual rate vector $\tilde{\mathbf{R}}^{\text {target }}$ sits on the boundary of $\mathcal{C}_{\mathrm{MAC}}\left(P, \mathbf{H}^{H}\right)$.

Each set of $\left\{q_{i}\right\}$ that satisfies the sum-power constraint with equality, i.e., $\sum_{i} q_{i}=P$, produces $K$ ! achievable-rate vectors, one for each unique decoding order. All linear combinations of these rate vectors are achievable, and, hence, the MAC capacity region with individual power constraints $\left\{q_{i}\right\}$ is a polyhedron with the $K$ ! rate vectors as its vertices. Let us denote this as $\mathcal{C}_{\text {MAC }}\left(\left\{q_{i}\right\}, \mathbf{H}^{H}\right)$. Then $\mathcal{C}_{\text {MAC }}\left(P, \mathbf{H}^{H}\right)$ is a union of all such regions for which $\sum_{i} q_{i}=P$, and each point on its boundary corresponds to a vertex of one MAC region $\mathcal{C}_{\mathrm{MAC}}\left(\left\{q_{i}\right\}, \mathbf{H}^{H}\right)$.

The MAC capacity region $\mathcal{C}_{\text {MAC }}\left(\left\{q_{i}\right\}, \mathbf{H}^{H}\right)$ has a very special geometric structure. Each of its faces is parallel to at least one of the $R_{i}$ axes, except for a single slanted face with a normal vector $(1,1, \ldots, 1)$. This slanted face has $K$ ! vertices, corresponding to the $K$ ! decoding orders. A key observation here is that each vertex of $\mathcal{C}_{\mathrm{MAC}}\left(\left\{q_{i}\right\}, \mathbf{H}^{H}\right)$ is the solution to the optimization problem

$$
\max _{\tilde{q}_{1}, \ldots, \tilde{q}_{K}} \sum_{i=1}^{K} \alpha_{i} R_{i}^{\mathrm{MAC}}\left(\boldsymbol{\pi},\left\{\tilde{q}_{j}\right\}\right) \quad \text { s.t. } \quad \tilde{q}_{j} \leq q_{j}
$$

where $R_{i}^{\mathrm{MAC}}\left(\boldsymbol{\pi},\left\{\tilde{q}_{j}\right\}\right)$ is the rate achievable by user $i$ using the power allocation $\left\{\tilde{q}_{j}\right\}$ and successive decoding in the order $\boldsymbol{\pi}$ such that

$$
\alpha_{\pi(1)} \leq \alpha_{\pi(2)} \leq \cdots \leq \alpha_{\pi(K)} .
$$

By cycling through all $K$ ! relative magnitudes of $\left\{\alpha_{i}\right\}$, all the vertices can be found. This observation has been made in [26], and the geometric structure has been studied in detail in [27].

Returning to our problem, note that the boundary points of $\mathcal{C}_{\mathrm{MAC}}\left(P, \mathbf{H}^{H}\right)$ can now be found by solving an optimization problem similar to (18)

$$
\max _{q_{1}, \ldots, q_{K}} \sum_{i=1}^{K} \alpha_{i} R_{i}^{\mathrm{MAC}}\left(\boldsymbol{\pi},\left\{q_{j}\right\}\right) \text { s.t. } \sum_{j=1}^{K} q_{j} \leq P .
$$

As in (18), $\boldsymbol{\pi}$ is determined by the relative magnitudes of $\left\{\alpha_{i}\right\}$ through (19). Varying the weights $\left\{\alpha_{i}\right\}$ [and correspondingly the decoding order $\boldsymbol{\pi}$ according to (19)] traces out the boundary of the entire capacity region. The optimal user-ordering problem is, therefore, solvable in the dual MAC, and, hence, the BC. The full algorithm is given in detail in the next section.

Further, it turns out that the optimal ordering (19) automatically gives rise to a weighted sum-rate maximization problem which has a convex structure. This is easily shown by expanding the objective function in (20) using (16) to yield

$$
f\left(q_{1}, \ldots, q_{K}\right)=\sum_{i=0}^{K-1}\left(\alpha_{\pi(i+1)}-\alpha_{\pi(i)}\right) \log \left(1+\sum_{j=i+1}^{K} q_{j}\left\|\mathbf{h}_{j}\right\|^{2}\right)
$$

where $\alpha_{\pi(0)}=0$ by convention. Since $\alpha_{\pi(i+1)} \geq \alpha_{\pi(i)}$, $f\left(q_{1}, \ldots, q_{K}\right)$ is a positively weighted sum of logarithms, its second derivative is negative and, hence, $f(\cdot)$ is concave in its arguments. Since the constraint $\sum_{i} q_{i} \leq P$ is convex, (20) with optimal ordering is a convex optimization problem.

To summarize, uplink-downlink duality tremendously simplifies the computation of the capacity region. The two key observations are: 1 ) the optimal ordering ${ }^{3}$ is determined by the relative magnitudes of the weights $\left\{\alpha_{i}\right\}$ prior to actually solving for the optimal rates; and 2) the optimal ordering gives rise to a convex optimization problem for the MAC channel. Neither of these statements directly holds for the $\mathrm{BC}$ capacity region. These two observations are the key enabling facts that make the MAC capacity region much easier to deal with than the BC capacity region.

\section{USER ORDERING}

\section{A. Power Minimization With Fixed Arbitrary Ordering}

When the encoding or decoding order $\boldsymbol{\pi}$ is fixed arbitrarily, the transmit-power minimization problem for the downlink (11) is easy to solve using duality, because user $\pi(K)$ achieves a rate of

$$
R_{\pi(K)}=\log \left(1+q_{\pi(K)} \mathbf{h}_{\pi(K)}^{H} \mathbf{h}_{\pi(K)}\right)
$$

which must equal $\tilde{R}_{\pi(K)}^{\text {target }}$. Therefore, $q_{\pi(K)}$ can be found as

$$
q_{\pi(K)}=\frac{1}{\mathbf{h}_{\pi(K)}^{H} \mathbf{h}_{\pi(K)}}\left(2^{\tilde{R}_{\pi(K)}^{\text {target }}}-1\right)
$$

Given $q_{\pi(K)}, q_{\pi(K-1)}$ can be found because the rate expression for user $\pi(K-1)$ only depends on $q_{\pi(K)}$ and $q_{\pi(K-1)}$. In fact, for user $\pi(i)$

$$
q_{\pi(i)}=\frac{2^{\tilde{R}_{\pi(i)}^{\text {target }}}-1}{\mathbf{h}_{\pi(i)}^{H}\left(\mathbf{I}+\sum_{j=i+1}^{K} q_{\pi(j)} \mathbf{h}_{\pi(j)} \mathbf{h}_{\pi(j)}^{H}\right)^{-1} \mathbf{h}_{\pi(i)}} .
$$

The set $\left\{q_{i}\right\}$ thus obtained ensures that $\tilde{\mathbf{R}}^{\text {target }}$ is on the boundary of the achievable-rate region for decoding order $\boldsymbol{\pi}$, and, hence, that total power $P$ is minimized. The transmit covariance matrices $\left\{\mathbf{S}_{i}\right\}$ for the downlink can now be obtained through the covariance transformations mentioned earlier [12]. It is easily shown that each $\mathbf{S}_{i}$ will be a rank-one matrix, or $\mathbf{S}_{i}=\lambda_{i} \mathbf{v}_{i} \mathbf{v}_{i}^{H}$, where $\lambda_{i}$ is the only nonzero eigenvalue of $\mathbf{S}_{i}$ and $\mathbf{v}_{i}$ is the associated unit-norm eigenvector. The vector $\mathbf{v}_{i}$ is

\footnotetext{
${ }^{3}$ In other words, the order that must be applied to reach the point on the boundary of $\mathcal{C}_{\mathrm{MAC}}\left(P, \mathbf{H}^{H}\right)$ that has $\sum_{i} \alpha_{i} R_{i}^{\mathrm{MAC}}=$ constant as its tangential plane.
} 


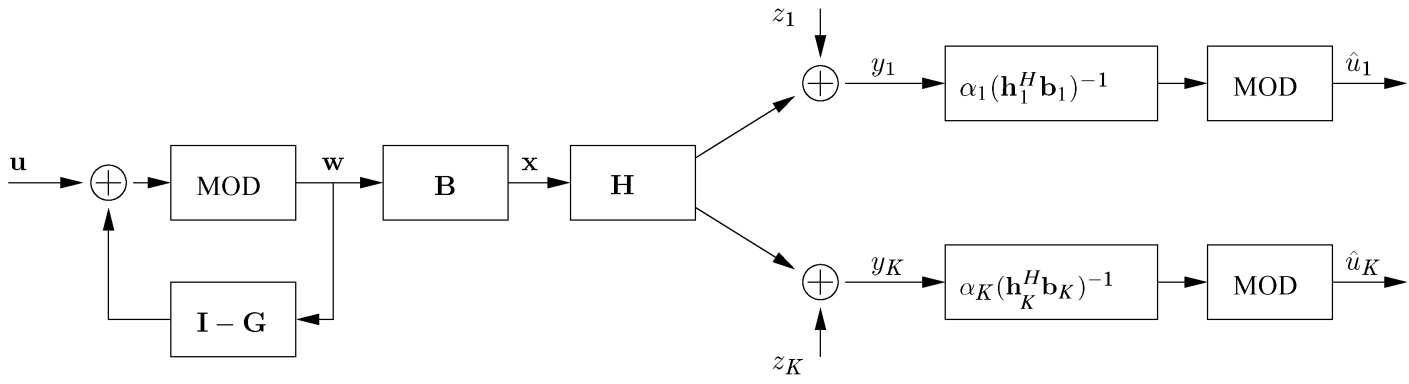

Fig. 1. Block diagram of the THP-based transmitter, channel, and individual receivers. $\mathbf{u}$ and $\mathbf{w}$ are $K$-vectors, $\mathbf{x}$ is a $t$-vector, $y_{k}$ 's and $z_{k}$ 's are scalars. The output of the modulo operation $\hat{u}_{k}$ goes into a hard decision for an estimation of the $k$ th QAM symbol in $\mathbf{u}$.

the transmit beamforming vector, and $\lambda_{i}$ is the optimal power allocation for user $i$.

We propose to implement successive DPC encoding approximately using a matrix form of THP, as shown in Fig. 1, by translating the set of $\mathbf{S}_{i}$ matrices obtained above into a compact representation involving precoding matrices $\mathbf{G}$ and $\mathbf{B}$. This is akin to the precoding structure implemented for achieving the sum capacity in [16].

The vector $\mathbf{u}=\left[u_{1}, \ldots, u_{K}\right]$ contains the information symbols for users 1 to $K$. Each $u_{i}$ is drawn from a square- or a cross-QAM constellation with a constellation size determined by the actual rate requirement $\mathbf{R}$. Without loss of generality, let the minimum distance of the constellation be 2 . Let $\left[-L_{i}, L_{i}\right)$ be the interval within which the QAM constellation is contained. For example, at 4 bits/channel use, the constellation size is 16-QAM. The coordinates of the constellation points are chosen from $\{ \pm 1, \pm 3\}$, and $L_{i}=4$.

The feedback matrix $\mathbf{I}-\mathbf{G}$ is a strictly upper triangular matrix. For a given encoding order, $\mathbf{I}-\mathbf{G}$ serves to presubtract anti-causal ${ }^{4}$ MUI. The presubtracted value then goes through a modulo operation, producing a vector $\mathbf{w}$, which can be computed successively, from the last user to the first, as

$$
w_{i}=u_{i}-\sum_{j=i+1}^{K} g_{i j} w_{j} \bmod \left(-L_{i}, L_{i}\right]
$$

where $g_{i j}$ is the $(i, j)$ th element of $\mathbf{G}$. The modulo operation is an implementation of a Tomlinson-Harashima precoder which reduces the transmit power while maintaining the distinguishability of constellation points. A similar modulo operation is implemented at the receiver to recover the transmitted symbol.

We now compute the covariance of $\mathbf{w}$. As $w_{i}$ 's are the output of the THP, they have approximately a uniform distribution in $\left(-L_{i}, L_{i}\right]$. (This applies to $w_{1}$ to $w_{K-1}$ only, as precoding is not applied to the $K$ th user.) Let $\sigma_{w i}^{2}$ denote the power of $w_{i}$. For uniform distribution, $\sigma_{w i}^{2}=L_{i}^{2} / 3$. Further, due to the modulo operation, $w_{i}$ and $w_{j}$ are approximately uncorrelated for $i \neq j$. So, $\mathbb{E}\left[w_{i} w_{j}^{*}\right]=\sigma_{w i}^{2} \delta_{i j}$, and $\mathbf{R}_{w}=\mathbb{E}\left[\mathbf{w w}^{H}\right]=\operatorname{diag}\left(\sigma_{w 1}^{2}, \ldots, \sigma_{w K}^{2}\right)$.

Referring to Fig. 1, the precoded symbols $\mathbf{w}$ are multiplied by $\mathbf{B}$. Thus, the transmitted signal $\mathbf{x}=\mathbf{B w}$ has a covariance

\footnotetext{
${ }^{4}$ In the sense of treating users 1 through $K$ as time indexes 1 through $K$. For user or "time" $i$, interference from users $i+1$ through $K$ are subtracted.
}

matrix $\mathbb{E}\left[\mathbf{x x}^{H}\right]=\mathbf{B R}_{w} \mathbf{B}^{H}$. Therefore, the transmit covariance matrix for user $i$ is $\sigma_{w i}^{2} \mathbf{b}_{i} \mathbf{b}_{i}^{H}$, and to make that equal to the $\mathbf{S}_{i}$ obtained from minimizing power under virtual rate constraints, we simply set

$$
\mathbf{b}_{i}=\frac{\sqrt{\lambda_{i}}}{\sigma_{w i}} \mathbf{v}_{i}
$$

It is now possible to specify $\mathbf{G}$. The matrix $\mathbf{G}$ is designed to cancel interference from previously encoded users. Interestingly, complete subtraction is not necessarily the best thing to do [20], [28]. The THP is analogous to the cancellation of precursor intersymbol interference (ISI) in a DFE. A ZF-DFE is, in general, inferior to an MMSE-DFE, which only subtracts most (but not all) of the interference.

To design $\mathbf{G}$, note that the received signal at the $i$ th receiver is given by

$$
y_{i}=\mathbf{h}_{i}^{H} \mathbf{b}_{i} w_{i}+\mathbf{h}_{i}^{H} \sum_{j \neq i} \mathbf{b}_{j} w_{j}+n_{i} .
$$

For complete interference presubtraction (CIP), we want

$$
\mathbf{h}_{i}^{H} \sum_{j=i+1}^{K} \mathbf{b}_{j} w_{j}=0 .
$$

Coupled with (25), this yields

$$
g_{i j}=\frac{\mathbf{h}_{i}^{H} \mathbf{b}_{j}}{\mathbf{h}_{i}^{H} \mathbf{b}_{i}}
$$

so $\mathbf{G}$ is known once $\mathbf{B}$ is obtained.

In an MMSE design, a partial interference presubtraction (PIP) is done. In this case

$$
g_{i j}=\eta_{i} \frac{\mathbf{h}_{i}^{H} \mathbf{b}_{j}}{\mathbf{h}_{i}^{H} \mathbf{b}_{i}}
$$

where $\eta_{i}$ is chosen to be the MMSE factor [14], [20]

$$
\eta_{i}=\frac{\sigma_{w i}^{2}\left|\mathbf{h}_{i}^{H} \mathbf{b}_{i}\right|^{2}}{1+\sum_{j=1}^{i} \sigma_{w j}^{2}\left|\mathbf{h}_{i}^{H} \mathbf{b}_{j}\right|^{2}} .
$$

By scaling the channel receiver output by the same factor $\eta_{i}$, the effective noise can be reduced at the receiver [20], [28]. PIP is 
superior to CIP at low SNR [20], analogous to an MMSE DFE. Note that $\eta_{i}=1$ for CIP.

The THP structure proposed in this section is similar to many existing ones in the literature [3], [4], [29], [30]. However, most conventional THPs are designed as a function of the channel matrix only, and do not allow the flexibility of designing the precoder as a function of user data-rate requirements. Data rate guarantees are typically achieved with power loading only (see, e.g., [4]). The ability to optimize both the THP structure and power allocation is a novel feature of the precoder presented in this paper. Further, conventional THPs use either a fixed precoding order or a precoding order determined by some minimum total variance criterion [30]. This paper tackles the optimal user-ordering problem directly based on per-user data rate and probability of error requirements, as shown in the next section.

\section{B. Optimal Ordering}

For a fixed encoding order in the BC, the method of the last section can be used to determine the power-minimizing $\mathbf{B}$ and $\mathbf{G}$ matrices. To find the optimal order which will result in $\tilde{\mathbf{R}}^{\text {target }}$ lying on the boundary of $\mathcal{C}_{\mathrm{BC}}(P, \mathbf{H})$, the brute-force method would be to repeat the procedure for all $K$ ! possible orders. Such an exhaustive search is intractable in practice for large $K$.

The main idea proposed here is based on the results developed in Section III-B. The first step is to translate the data rate and BER constraints into virtual rate constraints. Then, via uplink-downlink duality, the downlink design problem is reformulated as an uplink design problem. The uplink capacity region is much easier to find because the optimal user-ordering problem for the uplink channel is trivial and the capacity-region optimization problem admits a convex structure. More specifically, for a given set of weights $\boldsymbol{\alpha}$ and power constraint $P$, we may find one of the vertices of the MAC capacity region by maximizing $\sum_{i} \alpha_{i} R_{i}$ as stated in (20).

Conversely, to find the minimal total transmit power subject to a rate constraint, we may do the following. First, fix a total power, and vary $\boldsymbol{\alpha}$ to test whether the rate requirement is inside the capacity region or not. If so, the total power may be reduced, otherwise, it must be increased. A two-loop optimization procedure would then be able to find the minimal power subject to a rate constraint.

More precisely, the algorithm is as follows.

1) Initialization: Choose $\alpha \in \mathbb{R}_{+}^{K}$ and $P \in \mathbb{R}_{+}$arbitrarily.

2) Rate Maximization: Use an iterative constrained optimization algorithm to solve (20).

3) Iteration in $\boldsymbol{\alpha}$ : Compare rates obtained in maximization step with desired rates $\tilde{\mathbf{R}}^{\text {target }}$. For all $i$, update $\alpha_{i}$ using

$$
\alpha_{i} \leftarrow \alpha_{i}+\mu_{i}\left(\tilde{R}_{i}^{\text {target }}-R_{i}\right)
$$

where $\mu_{i}>0$ is a small step size, and $R_{i}$ is the $i$ th user's rate from the maximization step. Declare convergence when $\tilde{R}_{i}^{\text {target }}>R_{i}$ or $\tilde{R}_{i}^{\text {target }}<R_{i}$ for all $i$.

4) Iteration in $P$ : If, after convergence of the $\boldsymbol{\alpha}$ iterations, $\tilde{R}_{i}^{\text {target }}>R_{i}$, then total power is too low; conversely,

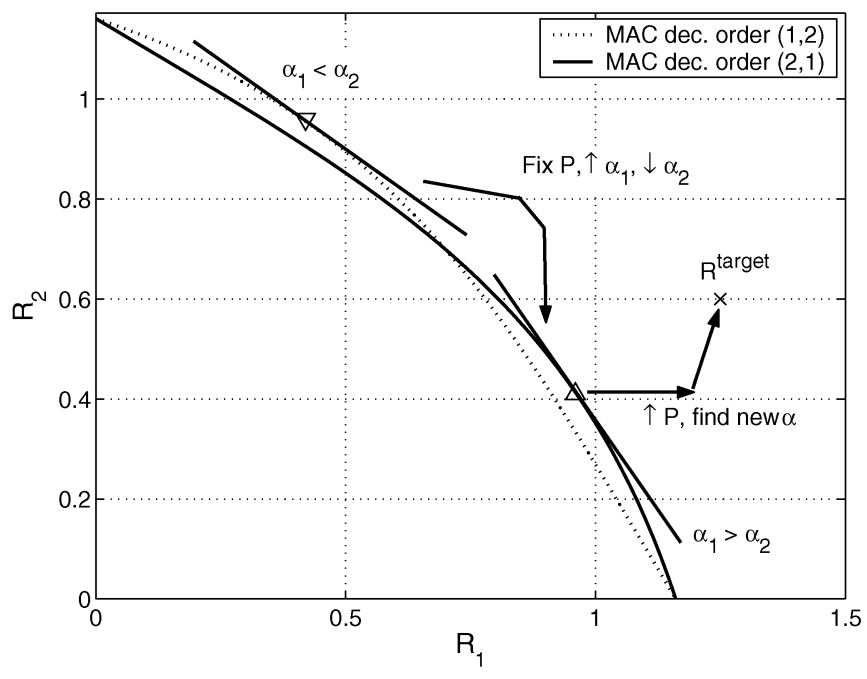

Fig. 2. Illustration of optimal encoding order algorithm.

if $\tilde{R}_{i}^{\text {target }}<R_{i}$, then total power is too high. So power constraint $P$ should be adjusted according to

$$
\begin{cases}P_{\min }=P & \text { if } \tilde{R}_{\dot{t}}^{\text {target }}>R_{i} \\ P_{\max }=P & \text { if } \tilde{R}_{i}^{\text {target }}<R_{i}\end{cases}
$$

and $P=\left(P_{\min }+P_{\max }\right) / 2$, where the initial $\left[P_{\min }, P_{\max }\right]$ is a preset allowable range of $P$.

5) Outer Iterations: Repeat steps $2-4$ until $\mathbf{R}=\tilde{\mathbf{R}}^{\text {target }}$.

We note that the iteration steps (32) and (33) are essentially subgradient updates on the dual variables associated with the rate constraints. Their convergence is guaranteed due to the convex structure of the optimization problem. However, in some instances, the inner iteration may enter a limit cycle as $\tilde{\mathbf{R}}^{\text {target }}$ could be on the dominant face of the capacity region, which requires time-sharing of the vertices. This can be easily detected in the implementation. The algorithm outlined above should persuade readers that it is possible to incorporate time-sharing into a full user-ordering algorithm.

Fig. 2 illustrates the proposed method. Consider a two-user system with given channel matrices. For a given initial $P$ and $\boldsymbol{\alpha}$ where $\alpha_{1}<\alpha_{2}$, we maximize $\alpha_{1} R_{1}+\alpha_{2} R_{2}$ assuming the MAC decoding order $1 \rightarrow 2$ (or BC encoding order $2 \rightarrow 1$ ) to arrive at the inverted triangle $\nabla$. The required virtual rate vector is marked by a cross $\times$ in the bottom right corner. Since at $\nabla$, $R_{1}$ is smaller than its target and $R_{2}$ is larger than its target, we adjust $\boldsymbol{\alpha}$ according to (32) by increasing $\alpha_{1}$ and decreasing $\alpha_{2}$. Maximizing $\alpha_{1} R_{1}+\alpha_{2} R_{2}$ again with the same $P$ and new $\boldsymbol{\alpha}$ brings us to $\triangle$, at which point, both $R_{1}$ and $R_{2}$ are smaller than their respective targets. This signals the convergence of the $\boldsymbol{\alpha}$ iterations, and that $P$ needs to be increased. After a number of steps of successive refinement of $\boldsymbol{\alpha}$ and $P$, we will arrive at the target point $\times$. The optimization process gives both the optimal encoding order and the required $\mathbf{B}$ and $\mathbf{G}$ matrices of the THP.

\section{Near-Optimal User Ordering}

The above optimal ordering algorithm, despite having a polynomial complexity, is still fairly complex to implement in practice, as it calls for a convex optimization problem to be solved repeatedly. The main result of this section is a heuristic, yet 
near-optimal, user-ordering algorithm with an even lower complexity.

We again use the key observation that the optimal encoding or decoding order for a target rate $\tilde{\mathbf{R}}^{\text {target }}$ must be such that if $\tilde{\mathbf{R}}^{\text {target }}$ is a solution to an uplink rate-maximization problem $\max \boldsymbol{\alpha}^{T} \mathbf{R}$, then the optimal order must be the one suggested by $\boldsymbol{\alpha}$. This property can be easily verified, and if it fails to hold for some particular order, then this order must not be the optimum.

The above observation gives us the following heuristic order update method. Choose some arbitrary order $\boldsymbol{\pi}$, find the weight vector $\boldsymbol{\alpha}$ for which $\tilde{\mathbf{R}}^{\text {target }}$ is the solution to the maximization of $\boldsymbol{\alpha}^{T} \mathbf{R}$, and compare the order suggested by $\boldsymbol{\alpha}$ with the order originally picked. If they are not the same, we may then replace the original ordering by the new order suggested by $\boldsymbol{\alpha}$.

The main outstanding issue in the above approach is that it is not easy to find the $\boldsymbol{\alpha}$ for which the given $\tilde{\mathbf{R}}^{\text {target }}$ is a solution to

$$
\max \boldsymbol{\alpha}^{\boldsymbol{T}} \mathbf{R} \quad \text { s.t. } \mathbf{R} \in \mathcal{C}_{\mathrm{BC}}(P, \mathbf{H}) .
$$

This is so because solving the above requires an iterative process in $\boldsymbol{\alpha}$. However, it is easy to identify the weight vector if the maximization problem is constrained to be of the fixed order

$$
\max \boldsymbol{\alpha}^{\boldsymbol{T}} \mathbf{R} \quad \text { s.t. } \mathbf{R} \in \mathcal{C}_{\mathrm{BC}}(P, \mathbf{H}, \boldsymbol{\pi}) .
$$

This is so because the rate region $\mathcal{C}_{\mathrm{BC}}(P, \mathbf{H}, \boldsymbol{\pi})$ has a specific form. In particular, by uplink-downlink duality, the region is exactly the same as $\mathcal{C}_{\mathrm{MAC}}\left(P, \mathbf{H}^{H}, \boldsymbol{\pi}\right)$. In the suboptimal algorithm, we opt to approximate the true weights by finding the $\boldsymbol{\alpha}$ for which $\tilde{\mathbf{R}}^{\text {target }}$ is the solution to the problem (35) instead of (34).

Such an $\boldsymbol{\alpha}$ may be identified as follows. The boundary of the rate region $\mathcal{C}_{\mathrm{MAC}}\left(P, \mathbf{H}^{H}, \boldsymbol{\pi}\right)$ is defined by a power constraint

$$
\sum_{i=1}^{K} q_{i} \leq P .
$$

Thus, the optimality condition for the optimization problem is

$$
\sum_{i=1}^{K} \alpha_{i} \frac{\partial R_{i}}{\partial q_{j}}=\lambda \quad \forall j
$$

where $\lambda$ is the Lagrangian multiplier associated with the power constraint. In a matrix form, $\alpha$ may be found by solving the following system of linear equations:

$$
\left[\begin{array}{cccc}
\frac{\partial R_{1}}{\partial q_{1}} & \frac{\partial R_{2}}{\partial q_{1}} & \cdots & \frac{\partial R_{K}}{\partial q_{1}} \\
\frac{\partial R_{1}}{\partial q_{2}} & \frac{\partial R_{2}}{\partial q_{2}} & \cdots & \frac{\partial R_{K}}{\partial q_{2}} \\
\vdots & \vdots & \ddots & \vdots \\
\frac{\partial R_{1}}{\partial q_{K}} & \frac{\partial R_{2}}{\partial q_{K}} & \cdots & \frac{\partial R_{K}}{\partial q_{K}}
\end{array}\right]\left[\begin{array}{c}
\alpha_{1} \\
\alpha_{2} \\
\vdots \\
\alpha_{K}
\end{array}\right]=\lambda\left[\begin{array}{c}
1 \\
1 \\
\vdots \\
1
\end{array}\right] .
$$

The partial derivative $\partial R_{j} / \partial q_{i}$ may be computed via the dualuplink rate region expression (16) at the power vector $\mathbf{q}$ found using the algorithm in Section IV-A. Note that $\boldsymbol{\alpha}$ may be scaled by a constant without affecting the relative ordering, and, hence, we can set $\lambda=1$ without loss of generality.

Geometrically, $\alpha$ is the normal vector of the hyperplane tangential to the boundary of $\mathcal{C}_{\mathrm{BC}}(P, \mathbf{H})=\mathcal{C}_{\mathrm{MAC}}\left(P, \mathbf{H}^{H}\right)$ at the

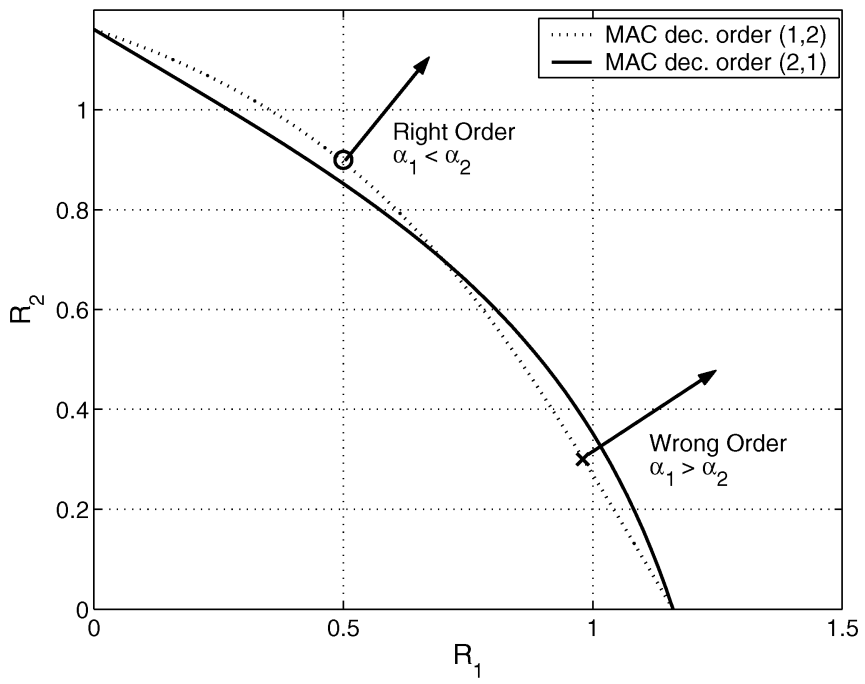

Fig. 3. Near-optimal user-ordering algorithm. If assumed order in the virtual MAC is $1 \rightarrow 2$, then $\alpha$ found using (38) will have $\alpha_{1}<\alpha_{2}$ if the target rate vector is at $\circ$, but $\alpha_{1}>\alpha_{2}$ if the target rate vector is at $\times$. In the first case, the values of $\alpha_{1}$ and $\alpha_{2}$ indicate the right order was assumed and the algorithm terminates; in the second case, we know the wrong order was assumed, so we try the order $2 \rightarrow 1$ indicated by the relative sizes of $\alpha_{1}$ and $\alpha_{2}$.

point $\mathbf{R}=\tilde{\mathbf{R}}^{\text {target }}$. The optimization process is equivalent to pushing the hyperplane $\alpha^{T} \mathbf{R}=\beta$ as far away from the origin as possible while still remaining within the capacity region. At the furthest point, $\beta=\boldsymbol{\alpha}^{T} \tilde{\mathbf{R}}^{\text {target }}$, and, hence, the hyperplane tangential to $\mathcal{C}_{\mathrm{MAC}}\left(P, \mathbf{H}^{H}\right)$ at $\mathbf{R}=\tilde{\mathbf{R}}^{\text {target }}$ is described by

$$
\boldsymbol{\alpha}^{\boldsymbol{T}}\left(\mathbf{R}-\tilde{\mathbf{R}}^{\text {target }}\right)=0 .
$$

Since this is a tangent to the boundary of $\mathcal{C}_{\mathrm{MAC}}\left(P, \mathbf{H}^{H}\right), \boldsymbol{\alpha}$ is the normal vector to the boundary at the target rate tuple. The relation between $\boldsymbol{\alpha}$ and the tangent hyperplane is illustrated in Fig. 3 .

To summarize, the suboptimal ordering algorithm runs as follows. Fix a set of target rates $\tilde{\mathbf{R}}^{\text {target }}$.

1) Initialize: Choose some arbitrary order $\boldsymbol{\pi}$.

2) Minimize power: Use the fixed-order algorithm of Section IV-A to minimize total transmit power under $\tilde{\mathbf{R}}^{\text {target }}$ and $\pi$

3) Identify normal vector: Find a vector $\boldsymbol{\alpha}$ normal to the hyperplane tangent to $\mathcal{C}_{\mathrm{BC}}(P, \mathbf{H}, \boldsymbol{\pi})$ at $\mathbf{R}$ by solving (38) with $\lambda=1$.

4) Update $\boldsymbol{\pi}$ : Let $\boldsymbol{\pi}^{\prime}$ be the order for which $\alpha_{\pi^{\prime}(1)} \leq \cdots \leq$ $\alpha_{\pi^{\prime}(K)}$. If $\boldsymbol{\pi}=\boldsymbol{\pi}^{\prime}$, then declare $\boldsymbol{\pi}$ to be the best order. Otherwise, set $\boldsymbol{\pi}=\boldsymbol{\pi}^{\prime}$, and return to Step 2 .

The above algorithm is suboptimal because of the approximation in computing $\boldsymbol{\alpha}$ [i.e., replacing (35) by (34)] and step 4 above, setting $\boldsymbol{\pi}$ to $\boldsymbol{\pi}^{\prime}$ at each iteration does not ensure convergence to the optimal order. In fact, it is possible for the algorithm to cycle through a finite number of orders, in which case, we may then use the best of the finite number of orders (in terms of total required power $P$ ) as the "near-optimal" order. Nevertheless, the simulation results in the next section show that the above ordering algorithm works well in practice. 


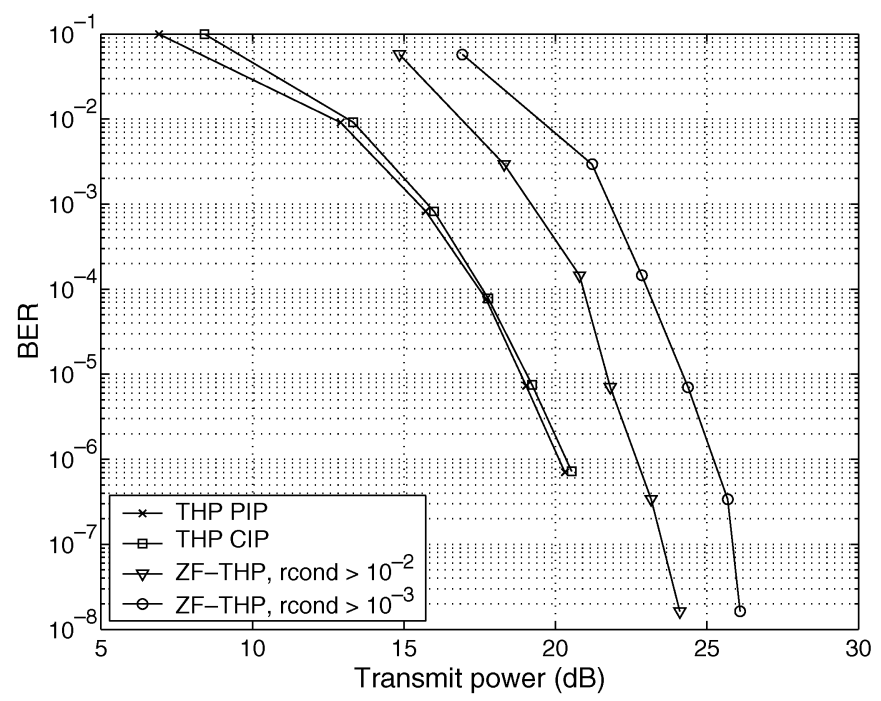

Fig. 4. BEP curves obtained through Monte Carlo simulations. Fixed arbitrary encoding order.

\section{NUMERICAL RESULTS}

In this section, we provide a number of comparisons between the methods proposed in this paper and competing schemes. In all simulations, we assume that there are four users $(K=4)$ and the base station has four antennas $\left(N_{t}=4\right)$. In Fig. 4 , the BER is plotted against total transmitted power $P$ for the two schemes proposed here. THP PIP stands for PIP, and THP CIP is obtained from complete presubtraction. These two curves were generated by changing the BER requirements from $10^{-1}$ down to $10^{-6}$ one decade at a time, and then using the fixed encoding order algorithm of Section IV-A to compute the required matrices in the THP transmitter. The BER averaged over all users through Monte Carlo simulations is then plotted against the resulting $P$. As expected, PIP outperforms CIP slightly. On the same figure are two additional curves, obtained by using the ZF-THP scheme of [4], which essentially solves the problem of cancelling all MUI while providing the required virtual rates to all users. This ZF scheme suffers from power enhancement at the transmitter, which can be significant when the channel is ill-conditioned. The two ZF curves in Fig. 4 correspond to the average BER, averaged over all channels with conditioning number larger than $10^{-2}$ and $10^{-3}$, respectively. Clearly, the precoding design proposed in this paper outperforms the $\mathrm{ZF}$ scheme.

To compare the various ordering schemes outlined in Sections IV-B and IV-C, Fig. 5 plots the target rate (equal for all users) against transmitted power $P$ for user orders obtained in five ways: 1) optimally (Section IV-B); 2) with the near-optimal algorithm of Section IV-C; 3) with the best (lowest power) order out of five randomly selected orders; 4) best of four randomly selected orders; and 5) a randomly selected order. At a desired rate of 2 bits per channel use per user, the difference between the arbitrary order and the optimal order is almost $2 \mathrm{~dB}$, but there is barely any noticeable difference between the optimal and near-optimal methods we proposed, in spite of the large difference in complexity between the two schemes. This shows that the heuristic scheme proposed in Section IV-C works well in practice.

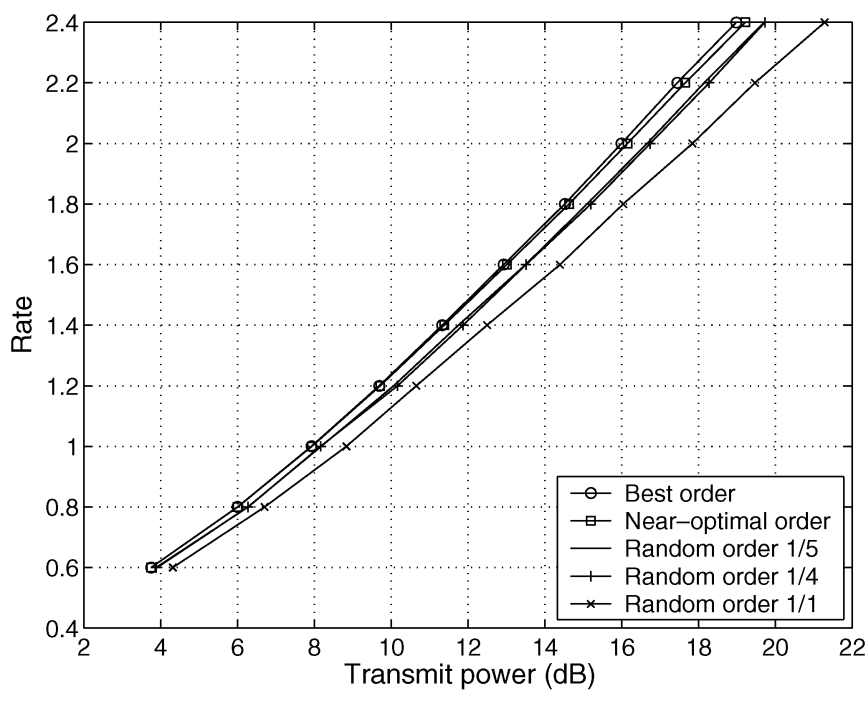

Fig. 5. Plots of target rates against transmitted power for various user-order methods. There are $4 !=24$ possible orders for this four-user system.

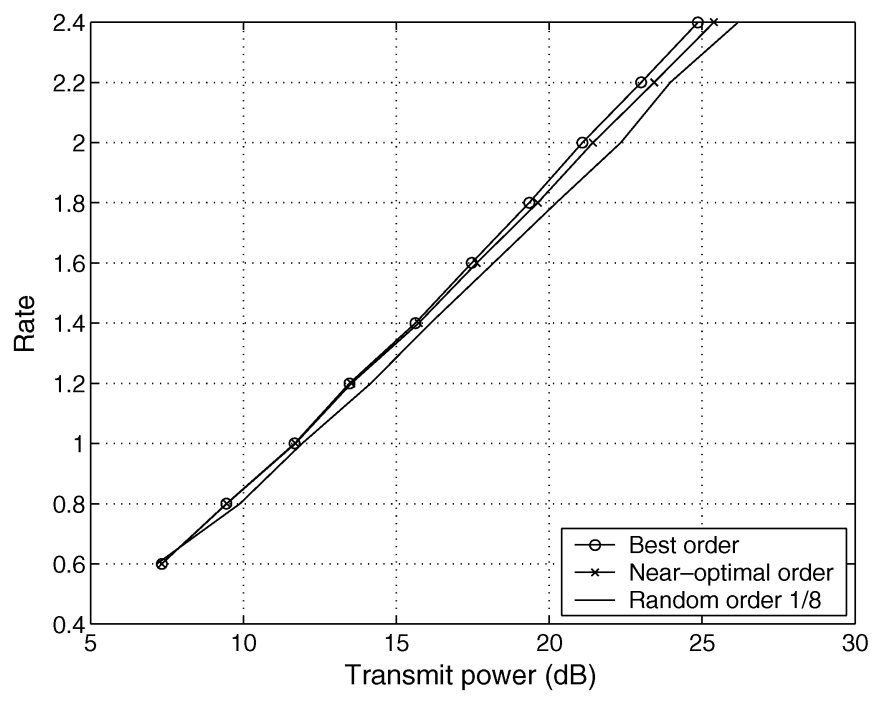

Fig. 6. Plots of target rates against transmitted power for various user-order methods. There are $6 !=720$ possible orders for this six-user system.

In Fig. 6, we show a plot similar to Fig. 5 in which the number of users has been increased to 6 , so that there are $6 !=720$ possible encoding orders. It is apparent that the near-optimal algorithm still performs very closely to the optimal algorithm.

\section{CONCLUSION}

This paper proposes a transmitter design for a downlink channel with multiple transmit antennas at the base station and a single receive antenna for each user. The design uses a Tomlinson-Harashima precoder at the transmitter which encodes each user's data successively, accounting for the interference at each receiver due to users already encoded. The proposed design minimizes the total transmit power necessary to deliver the required data rates to all users, at the BEPs required by each user. This is achieved using the novel concept of virtual rate, which accounts for practical modulation and THP schemes under a BER requirement, and an uplink-downlink duality result for capacity regions. The use of the virtual uplink makes 
it possible to take advantage of the geometric structure of the uplink capacity region in deriving the optimal user ordering, i.e., the one that requires minimum total power, with polynomial complexity. Finally, the insights gained from the optimal ordering scheme also allow a heuristic, but low-complexity and near-optimal, ordering algorithm to be derived.

\section{REFERENCES}

[1] B. R. Vojcic and W. M. Jang, "Transmitter precoding in synchronous multiuser communications," IEEE Trans. Commun., vol. 46, no. 10, pp. 1346-1355, Oct. 1998.

[2] Q. H. Spencer, A. L. Swindlehurst, and M. Haardt, "Zero-forcing methods for downlink spatial multiplexing in multiuser MIMO channels," IEEE Trans. Signal Process., vol. 52, no. 2, pp. 461-471, Feb. 2004.

[3] G. Ginis and J. M. Cioffi, "Vectored transmission for digital subscriber line systems," IEEE J. Sel. Areas Commun., vol. 20, pp. 1085-1104, Jun. 2002.

[4] C. Windpassinger, R. F. H. Fischer, T. Vencel, and J. B. Huber, "Precoding in multi-antenna and multi-user communications," IEEE Trans. Wireless Commun., vol. 3, no. 4, pp. 1305-1316, Jul. 2004.

[5] F. Rashid-Farrokhi, K. J. R. Liu, and L. Tassiulas, "Transmit beamforming and power control for cellular wireless systems," IEEE J. Sel. Areas Commun., vol. 16, no. 8, pp. 1437-1450, Oct. 1998.

[6] E. Visotsky and U. Madhow, "Optimum beamforming using transmit antenna arrays," in Proc. IEEE Vehicular Technol. Conf., Houston, TX, May 1999, vol. 1, pp. 851-856.

[7] M. Schubert and H. Boche, "Solution of the multiuser downlink beamforming problem with individual SINR constraints," IEEE Trans. Veh. Technol., vol. 53, no. 1, pp. 18-28, Jan. 2004.

[8] — "Solution of the multiuser downlink beamforming problem with individual SINR constraints," IEEE Trans. Signal Process., vol. 57, no. 7, pp. 2324-2334, Jul. 2005.

[9] J. Chang, L. Tassiulas, and F. Rashid-Farrokhi, "Joint transmitter-receiver diversity for efficient space division multiaccess," IEEE Trans. Wireless Commun., vol. 1, no. 1, pp. 16-27, Jan. 2002.

[10] Q. H. Spencer and A. L. Swindlehurst, "A hybrid approach to spatial multiplexing in multiuser MIMO downlinks," EURASIP J. Wireless Commun. Netw., vol. 2004, no. 2, pp. 236-247.

[11] R. Doostnejad, T. J. Lim, and E. S. Sousa, "Precoding for the MIMO broadcast channels with multiple antennas at each receiver," in Proc. 39th Conf. Inf. Sci. Syst., Baltimore, MD, Mar. 2005, CD-ROM.

[12] S. Vishwanath, N. Jindal, and A. Goldsmith, "Duality, achievable rates, and sum-rate capacity of Gaussian MIMO broadcast channels," IEEE Trans. Inf. Theory, vol. 49, no. 10, pp. 2658-2668, Oct. 2003.

[13] P. Viswanath and D. Tse, "Sum capacity of the vector Gaussian broadcast channel and uplink-downlink duality," IEEE Trans. Inf. Theory, vol. 49, no. 8, pp. 1912-1921, Aug. 2003.

[14] M. Costa, "Writing on dirty paper," IEEE Trans. Inf. Theory, vol. IT-29, no. 3, pp. 439-441, May 1983.

[15] G. Caire and S. Shamai, "On the achievable throughput of a multiantenna Gaussian broadcast channel," IEEE Trans. Inf. Theory, vol. 49, no. 7, pp. 1691-1706, Jul. 2003.

[16] W. Yu and J. M. Cioffi, "Sum capacity of Gaussian vector broadcast channels," IEEE Trans. Inf. Theory, vol. 50, no. 9, pp. 1875-1892, Sep. 2004.

[17] H. Weingarten, Y. Steinberg, and S. Shamai, "The capacity region of the Gaussian multiple-input-multiple-output broadcast channels," IEEE Trans. Inf. Theory, vol. 52, no. 9, pp. 3936-3964, Sep. 2006.

[18] M. Tomlinson, "New automatic equalizer employing modulo arithmetic," Electron. Lett., vol. 7, pp. 138-139, Mar. 1971.

[19] M. Miyakawa and H. Harashima, "A method of code conversion for a digital communication channel with intersymbol interference," Trans. Inst. Elect. Commun. Eng. Japan, vol. 52-A, pp. 272-273, Jun. 1969.

[20] W. Yu, D. P. Varodayan, and J. M. Cioffi, "Trellis and convolution precoding for transmitter-based interference presubtraction," IEEE Trans. Commun., vol. 53, no. 7, pp. 1220-1230, Jul. 2005.

[21] R. F. H. Fischer, Precoding and Signal Shaping for Digital Transmission. New York: Wiley-IEEE Press, 2002.

[22] J. M. Cioffi, Digital Communication. Stanford, CA: Stanford Univ., 2004, Lecture Notes.

[23] J. R. Barry, E. A. Lee, and D. G. Messerschmitt, Digital Communication, 3rd ed. Norwell, MA: Kluwer, 2004.

[24] S. Shamai and R. Laroia, "The intersymbol interference channel: Lower bounds on capacity and channel precoding loss," IEEE Trans. Inf. Theory, vol. 42, no. 5, pp. 1388-1404, Sep. 1996.
[25] W. Yu, "Uplink-downlink duality via minimax duality," IEEE Trans. Inf. Theory, vol. 52, no. 2, p. 2, Feb. 2006.

[26] R. S. Cheng and S. Verdú, "Gaussian multiaccess channels with ISI: Capacity region and multiuser water-filling," IEEE Trans. Inf. Theory, vol. 39, no. 3, pp. 773-785, May 1993.

[27] D. Tse and S. Hanly, "Multi-access fading channels-Part I: Polymatroid structure, optimal resource allocation and throughput capacities,' IEEE Trans. Inf. Theory, vol. 44, no. 7, pp. 2796-2815, Nov. 1998.

[28] R. Zamir, S. Shamai, and U. Erez, "Nested linear/lattice codes for structured multiterminal binning," IEEE Trans. Inf. Theory, vol. 48, no. 6 , pp. 1250-1276, Jun. 2002.

[29] O. Simeone, Y. Bar-Ness, and U. Spagnolini, "Linear and nonlinear preequalization/equalization for MIMO systems with long-term channel state information at the transmitter," IEEE Trans. Wireless Commun., vol. 3, no. 2, pp. 373-378, Mar. 2004.

[30] J. Liu and W. A. Krzymien, "Signal detection and pre-processing algorithms in layered space-time multiple input multiple output antenna systems," in Proc. Wireless Conf., Calgary, AB, Canada, Jul. 2004, pp. $9-21$.

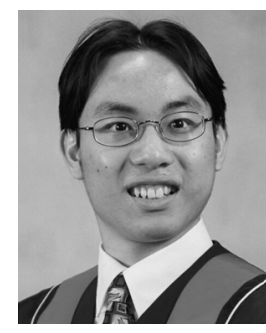

Chi-Hang Fred Fung (S'98) received the B.A.Sc. degree in electrical engineering from the University of Waterloo, Waterloo, ON, Canada, in 2002, and the M.A.Sc. degree in electrical and computer engineering in 2004 from the University of Toronto, Toronto, ON, Canada, where he is currently working toward the Ph.D. degree in electrical and computer engineering.

His research interests include quantum key distribution, quantum information theory, and wireless communications.

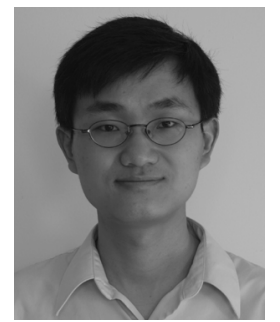

Wei Yu (S'97-M'02) received the B.A.Sc. degree in computer engineering and mathematics from the University of Waterloo, Waterloo, ON, Canada, in 1997 , and the M.S. and Ph.D. degrees in electrical engineering from Stanford University, Stanford, CA, in 1998 and 2002, respectively.

Since 2002, he has been an Assistant Professor with the Electrical and Computer Engineering Department, University of Toronto, Toronto, ON, Canada, where he also holds a Canada Research Chair. His main research interests are multiuser information theory, optimization, wireless communications and broadband access networks.

Prof. Yu is an Editor for the IEEE TRANSACTIONS ON WIRELESS COMmunications. He was a Guest Editor of a special issue of the IEEE JouRnal on SElected AREAS IN COMMUNICATIONS on Nonlinear Optimization of Communications Systems, and a Guest Editor of a special issue of the EURASIP Journal on Applied Signal Processing on Advanced Signal Processing for Digital Subscriber Lines.

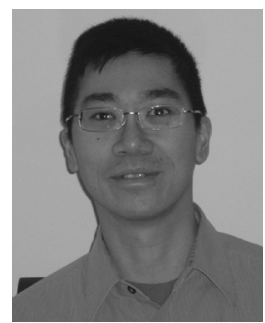

Teng Joon Lim (S'92-M'95-SM'02) received the B.Eng. degree from the National University of Singapore in 1992 and the Ph.D. degree from the University of Cambridge, Cambridge, U.K., in 1996, both in electrical engineering.

From September 1995 to November 2000, he was a Member of Technical Staff at the Centre for Wireless Communications in Singapore, now part of the Institute for Infocomm Research (I2R), where he held the position of Digital Communications Strategic Research Group Leader at the time of his departure. Since December 2000, he has been with the Electrical and Computer Engineering Department, University of Toronto, Toronto, ON, Canada, first as an Assistant Professor and now as an Associate Professor. He makes regular research contributions in a broad span of topics in wireless communications, such as multiuser detection and decoding, OFDM detection and synchronization, multiuser MIMO precoding, and cooperative diversity.

Prof. Lim has served on the technical program committees of several conferences, is an Associate Editor for the IEEE TRANSACTIONS ON VEHICULAR TECHNOLOGY, and is the vice-chair of the IEEE Toronto Communications Society chapter. He is a registered Professional Engineer in the province of Ontario. 